\title{
36
}

\section{Future environments}

\author{
J. F. Nunamaker, University of Arizona, Tucson Arizona USA, Chair
}

\section{PREAMBLE}

Given the high rate of change in technology and working environments, organizations are under tremendous pressure to improve their effectiveness. Many large organizations have evolved to the point where their structure and procedures are no longer in keeping with the needs of the future. Globalization of business, internationalization of trade and the increasing prevalence of multi-cultural interdisciplinary teams are beginning to redefine the nature of office work in general and decision making in particular. Organizations need to study themselves to determine what should be changed. Recognition must particularly be given to relationships between the organization and its external environment. The International Office of the Future (IOF) will be a dramatically different environment than that which exists in the majority of today's organizations. Imagine a meeting in the IOF:

\section{A SCENARIO}

As the participants arrive at their local conference room, the walls near the conference table light up with live video images from similar meeting sites at the location of the other participants. Camera angles and directional speakers give the illusion that the local conference table extends into the screen to the other sites, joining with the other tables forming a large single conference environment. Participants feel as if they are all present in the same room. They are able to converse with other group members and join various conversations at the other sites. Following introductions, the group leader assisted by a facilitator presents a decision making agenda that includes phases of problem framing, creative brainstorming, information organization, consensus formation and generation of action plans to achieve a value-added partnership complete with associated contractual obligations. Techniques and protocols are introduced and agreed upon to assist in managing the meeting process. The facilitator not only makes sure that the various cultures are respected but helps the group use its collective diversity to create a climate of creativity, synergism, and efficiency.

Group members are invited to present their opinions and participate in the discussions verbally as well as through use of a wide variety of technology interfaces including personal notepads and conventional laptop computers using electronic pens and voice recognition in addition to keyboards. Videowalls enable gestures and body language or remote participants to be recognized and acknowledged. Language translation is electronically supported, if needed, at least to the extent that exchanges are understandable, if not in the best grammatical form. 
A wide variety of software tools are available to support group members. For example, an "electronic brainstorming" tool provides each group member with an electronic copy of a common question. Ideas entered by participants are simultaneously shared with other group members. The result is that everyone is able to electronically speak at once and participate in multiple conversations. Group members can also access a variety of external data sources and expertise. Electronic "agents" directed by group members seek out relevant information that may bear on the topic at hand.

As the meeting continues, artificial intelligence is used to cluster and display common elements and streams of thought. As appropriate, analytical models and simulations are introduced to assist in negotiation and decision making. At various times during the meeting, group members make presentations or draw attention to a particular set of issues. Other group members are free to capture public information at their own computer, annotate visuals, and save information for their personal use as well as comment verbally and electronically back to the group and presenter as appropriate. Group members can vote electronically and begin to see consensus emerge as well as get personal feedback on their level of agreement with the group.

As the meeting draws to a close, some participants record on diskette information that they want to personally retain. Other participants send meeting information directly to their office computers. In addition, public information is stored in a team memory to be accessed and used by other stakeholders and in future meetings. Action plans are electronically circulated for comment and implementation is initiated as appropriate. Participants leave the meeting with a sense of accomplishment, commitment, and personal satisfaction in the negotiation and decision making process and product.

As participants return back to their offices, the issues from the meeting and action plans continue to move forward as other stakeholders take responsibility with a sense of purpose and direction as appropriate. Team members stay in touch through electronic mail, bulletin boards, electronic "scoreboards", desktop videoconferencing, and customized shared databases. As appropriate, subgroups as well as the main group reconvene to summarize progress, redirect energies, and conclude phases of larger projects.

\section{PANEL AIM}

For the most part, the technological components of this multi-dimensional environment exist to be integrated. However, without benefit of organizational development and behavioral wisdom, technology will be wasted at best and most likely counter-productive.

The challenge exists to sensibly integrate technological and organizational components. The key is a reasoned mix of theory and application through establishment of environments that meet the needs of the future. The IOF is a space, not just a place. This panel will explore issues associated with this multi-faceted domain. 